\title{
Severe anaphylaxis after general anesthesia induction
}

\author{
Yuan Sun ${ }^{1} \cdot$ Xiao Huang $^{2}$ (D) \\ Received: 5 June 2021 / Accepted: 19 June 2021 / Published online: 23 June 2021 \\ (c) Japanese Society of Anesthesiologists 2021
}

\section{To the Editor:}

We read the article by Horiuchi et al. [1] with interest. They have reported the data on the agents that cause anaphylaxis during general anesthesia. We commend the writers on their excellent research, which also has positive implications for the future in-depth understanding of drug-induced anaphylaxis. We would like to await additional studies to verify the conclusion by Horiuchi et al. The authors divided the patients into five groups by causative agents to study the characteristics of anaphylaxis. However, the degree of anaphylaxis can range in severity from modest objective breathing issues (such as moderate wheeze) to circulatory "shock" and/or collapse ("anaphylactic shock"). Severe anaphylaxis after anesthesia induction is uncommon in clinical practice, but it can be life-threatening. It might be meaningful to group patients according to the severity of the anaphylaxis. Besides, perioperative anaphylaxis usually occurs urgently and is therefore difficult to diagnose in time. It might be significant to clarify the diagnosis, treatment and prognosis of drug-induced anaphylaxis during general anesthesia.

Severe anaphylaxis is defined as a potentially lifethreatening impairment of the airway, breathing, and/or circulation, which can develop without the presence of skin reaction. These patients may not have a history of allergies. Some patients manifested with only unsustainable hypotension, or with unexplained elevated airway pressure. It is difficult for anesthesiologists to avoid severe anaphylaxis, especially in patients with no pre-operative history of allergy. The diagnosis of anaphylaxis during anesthesia is

Xiao Huang

huanghuang94@yeah.net

1 Department of Pharmacy, Beijing Chao-Yang Hospital, Capital Medical University, No. 8 of south worker's Stadium Road, Chao-Yang District, Beijing 100020, China

2 Department of Anesthesiology, Beijing Chao-Yang Hospital, Capital Medical University, No. 8 of south worker's Stadium Road, Chao-Yang District, Beijing 100020, China challenging because of the multiple drugs infused intravenously over a short period and the changes in the patient's clinical signs.

It is estimated that anaphylaxis occurs in one out of every 7,000 to 10,000 anesthesia cases, which may be higher in real data [2]. For some less qualified anesthesiologists facing sudden unanticipated severe anaphylaxis, how to deal with it in a short time is a problem. The general principles of anaphylaxis during anesthesia are guided by suspected allergens removal, airway control, Trendelenbourg position, epinephrine, glucocorticoids, and 24-h monitoring [3]. Although the theoretical knowledge describes the principle of treatment, how to respond to it immediately to reduce adverse reactions and improve prognosis is a challenge. It is necessary to understand the severity of anaphylaxis. Current research on perioperative anaphylaxis is limited to retrospective studies and case reports. There is a lack of large prospective studies about that. In addition, studies focusing on severe anaphylaxis after general anesthesia induction are lacking.

\section{References}

1. Horiuchi T, Takazawa T, Orihara M, Sakamoto S, Nagumo K, Saito S. Drug-induced anaphylaxis during general anesthesia in 14 tertiary hospitals in Japan: a retrospective, multicenter, observational study. J Anesth. 2021;35(1):154-60.

2. Harper NJN, Cook TM, Garcez T, Farmer L, Floss K, Marinho S, Torevell H, Warner A, Ferguson K, Hitchman J, Egner W, Kemp H, Thomas M, Lucas DN, Nasser S, Karanam S, Kong KL, Farooque S, Bellamy M, McGuire N. Anaesthesia, surgery, and life-threatening anaphylaxis: epidemiology and clinical features of perioperative anaphylaxis in the 6th National Audit Project (NAP6). Br J Anaesth. 2018;121(1):159-71.

3. Gouel-Chéron A, Harpan A, Mertes PM, Longrois D. Management of anaphylactic shock in the operating room. Presse Med. 2016;45(9):774-83.

Publisher's Note Springer Nature remains neutral with regard to jurisdictional claims in published maps and institutional affiliations. 